\title{
Privacy Analysis of Android Apps: Implicit Flows and Quantitative Analysis
}

\author{
Gianluca Barbon ${ }^{1}$, Agostino Cortesi ${ }^{1(\bowtie)}{ }^{(}$Pietro Ferrara $^{2}$, Marco Pistoia ${ }^{2}$, \\ and Omer Tripp ${ }^{2}$ \\ 1 Università Cá Foscari Venezia, Venice, Italy \\ cortesi@unive.it \\ 2 IBM Thomas J.Watson Research Center, Yorktown Heights, USA
}

\begin{abstract}
A static analysis is presented, based on the theory of abstract interpretation, for verifying privacy policy compliance by mobile applications. This includes instances where, for example, the application releases the user's location or device ID without authorization. It properly extends previous work on datacentric semantics for verification of privacy policy compliance by mobile applications by (i) tracking implicit information flow, and (ii) performing a quantitative analysis of information leakage. This yields to a novel combination of qualitative and quantitative analyses of information flows in mobile applications.
\end{abstract}

\section{Introduction}

Security threats are increasing in the mobile space, in particular in the Android environment. Specifically, mobile devices contain different sorts of confidential information that software might access. Such information is usually protected by permissions. However, the solutions provided by current mobile operating systems are not satisfying, and expose the user to various threats [8]. In addition, various applications and (e.g., analytics and advertisement) libraries make use of and sometimes leak user confidential data. Mobile security is also a major concern in an enterprise environment, where firms allow the use of company applications in the employee's personal device, increasing the risk of leakage of confidential business data. Therefore, there is and increasing request and need to formally verify the behavior of mobile applications, and to assess (and possibly limit) the quantity of released data. On the opposite side, a complete absence of data leakage of data would compromise the functionalities of mobile software. For instance, a navigation app like Waze needs to access the user location and communicate it to its servers in order to show appropriate traffic information. However, the user might want to prevent to leakage of her location to the advertisement engine. Ideally, we would like to impose — via suitable privacy policies — constraints on levels of data release, and give the user better awareness of the direct and indirect actual information flow concerning her personal data.

In this context, current research follows two main approaches: a statistical one $[14,17]$ and a language based one (e.g., information flow taint analysis) $[1,11,21-24]$. Both approaches suffer some weaknesses: the former does not

(C) IFIP International Federation for Information Processing 2015

K. Saeed and W. Homenda (Eds.): CISIM 2015, LNCS 9339, pp. 3-23, 2015.

DOI: $10.1007 / 978-3-319-24369-6 \_1$ 
fit well for qualitative analysis, while the latter as it is too strict, due to the fact that the non-interference notion [7] yields too many false positives limiting the effectiveness of the analysis.

In this paper, we extend our previous work [4]. This includes two primary contributions: (i) we investigate also implicit flows, where previously we considered only direct information release paths, and (ii) we relate explicit and implicit information flow to a quantitative notion of information leakage. We formalize our approach in the abstract interpretation framework. The advantage of such a method is that it enabes a general abstraction of all possible executions of a given program. Therefore, following the abstract-interpretation framework [6], we design an enhanced concrete semantics that formalizes how the expressions generated by the program's execution maintain footprints of (possibly confidential) data stored in the local data-store of the mobile device. With this formalization of the concrete semantics, we show how to create a sound abstraction such that the analysis is computable.

This work leads to the definition of a framework that merges quantitative and qualitative approaches by taking advantage of their respective strengths. We exploited the evaluation of single operators for the former approach and the collection of quantities of released information for the latter. Last, but certainly not least, the definition of this method has revealed the important role of the implicit flow in the leakage of secret variables. We evaluated the effectiveness of this framework over some benchmark examples.

The paper is structured as follows. After a brief introduction that describes related research and fundamental notions in Section 2, Section 3 recalls the semantics introduced by Cortesi et al. [4] extending it to capture implicit flows as well. Section 4 introduces the new quantitative approach that is added to the semantics described in the previous Sections. Section 5 introduces an abstraction of the quantitative analysis. Finally, in Section 6 a few significant examples of real working applications are presented and analyzed. Section 7 concludes.

\section{Background}

This Section introduces some important notions that will be used throughout the rest of the paper, and briefly describes the current related research.

Implicit Flows. Implicit flows were described by Denning [7] in 1976. Implicit flows have origins from the so called control statements, like if and while statements, where they are generated by their conditional expression. For instance, consider the following example:

if $b$ then $x=0$ else $x=1$;

Even if the final value of $x$ does not allows to directly recover the value of $b$, the latter affects the value of $x$, and an indirect information flow occurs from $b$ to $x$. Of course, implicit flows may yield to malicious effects[19]. 
Quantitative Approaches. A quantitative approach tracks some quantity, or measure, of leaked information. In [17] a new technique is proposed for determining the quantity of sensitive information that is revealed to public. The main idea presented by the authors consists in computing a maximum flow between the program inputs and the outputs, and by setting a limit on the maximum quantity of information revealed. The information flow is measured using a sort of network flow capacity, where the maximum rate of an imaginary fluid into this network represents the maximum extent of revealed confidential information. This method requires a dynamic approach in order to construct the graph, by performing multiple runs of the target program.

Quantitative Value Expressed as Bits. McCamant et al. [17] introduced a quantity concept in order to measure bits of information that can be released by the observation of a specific execution of a program. One of the first attempts to quantify information flow is the one of Lowe [14]. The author described quantity as number of bits, and defined the information flow as information passing between an high level user and a low level user through a covert channel. An interesting feature presented in this work consists in the assignment of 1 bit of quantity also with absence of information flow. This means that the author considers the absence of information as having value 1 bit. Finally they also introduce a time notion in the flow analysis. Another interesting approach is the one by Clark et al.[2,3]. First, they analyse $k$ bit variables, where $2^{k}$ are the values that can be represented from such variables. Second, they relate the maximum content of a variable to its data type, and they consider this as the possible quantity of leakage. Finally, they define the difference between the quantity of information of a confidential input and the amount of leaked information.

Security in Mobile Environments. Nowadays smartphones are used to store, modify and collect private and confidential data, e.g. location data or phone identifier. At the same time, a lot of malicious applications able to stole data or to track users exist. Mobile operating systems are not able to grant to users an appropriate control over confidential data and on how applications manage such data [8]. These limits make these platforms a potential target for attackers. An evolution of threats in mobile environments has been stressed also by the MacAfee Labs Threats Report [15]; in particular it underlines the existence of untrusted marketplaces and the increasing diffusion of open-source and commercial mobile malware source code, that facilitate the creation of such threats by unskilled attackers. Among mobile operating systems, Android is currently the most prevalent one [13], thus becoming the target of various threats. This mobile environment present different vulnerabilities. First, there is a lack of common definitions for securities and a high volume of available applications that guarantees the diffusion of malicious programs [9]. Second, many applications make use of private information, like the IMEI (International Mobile Station Equipment Identity), and of advertising and analytic libraries, that sends user data to remote servers for profiling. Third, the opportunity to install also applications 
coming from untrusted marketplaces makes the verification of these applications harder [18]. Last, but not least, even if Android requests to grant permission in order to install an application, this kind of control is not sufficient to avoid undesirable behaviour, because restrictions are not fine-grained [10,12].

Confidentiality Analysis in Mobile Environments. The importance of confidentiality analysis is growing in recent years, especially in the mobile space. In this field two main approaches can be found: dynamic and static analysis methods. Among the works that use the former method we can find those regarding the evaluation of permission-hungry mobile applications [1,18]. In particular, the work of Enck et al. [8] presents TaintDroid, a tool that monitors sensitive data by using real time tracking, avoiding the needing to get access to the applications source code. The main idea consists in tracking sensitive data that flows through systems interfaces, used by applications to get access to local data. This approach has some limitations. For instance, it does not allow the tracking of control flows, and generates false positives. Another approach is the one of Hornyack et al. with AppFence [12], which imposes privacy controls by retrofitting the Android environment, without the need to modify applications. Yet another approach, by Tripp and Rubin [25], is to reason about information release in terms of data values, rather than data flow, where the judgment is based on value similarity measures fed into a Bayesian learning algorithm. However, dynamic approaches present some weaknesses. First, they fail to discover some malicious behaviour, because applications have learned to recognize analysis during execution [10]. Second, the majority of dynamic approaches uses coarse-grained approximations that lead to false alarms and also missed leaks [1], while on the contrary static ones are able to discover potential leaks before the execution of the analysed application.

\section{Collecting Semantics}

In this section we introduce the collecting semantics, that consists in the first fundamental step of our framework design. We define the syntax, the domains, and the semantics with a specific focus on control statements.

\subsection{Syntax}

The formalization is focused onthree types of data: strings $(s \in \mathbb{S})$, integers $(n \in \mathbb{Z})$ and Booleans $(b \in \mathbb{B})$. sexp, nexp, and bexp denotes string, integer, and Boolean expressions, respectively. $\ell$ is used to represent data-store entries, and lexp denotes label expressions. For instance, string expressions are defined by: $\operatorname{sexp}::=s\left|\operatorname{sexp}_{1} \circ \operatorname{sexp}_{2}\right| \operatorname{encrypt}(\operatorname{sexp}, k)\left|\operatorname{sub}\left(\operatorname{sexp}, n \exp _{1}, \exp _{2}\right)\right|$ hash $(\operatorname{sexp}) \mid \operatorname{read}(\operatorname{lexp})$, where o represents concatenation, encrypt the encryption of a string with a key $k, s u b$ the substring of $\operatorname{sexp}$ between $n_{1}$ and $n_{2}$, hash the computation of the hash value, and read the function that returns the value in the data-store that corresponds to the given label. 


\subsection{Domain}

By adexp we denote an atomic data expression that tracks the data sources of a specific value. Formally, an atomic data expression adexp is a set of elements $\left\langle\ell_{i},\left\{\left(o p_{j}, l_{j}^{\prime}\right): j \in J\right\}\right\rangle$, representing that the datum corresponding to label $\ell_{i}$ has been combined with data corresponding to labels $\ell_{j}^{\prime}$ through operators $o p_{j}$ to get the actual value of the expression.

The set of atomic data expressions is defined by: $\mathbb{D}=\left\{\left\langle\ell_{i}, L_{i}\right\rangle: i \in I \subseteq\right.$ $\left.\mathbb{N}, \ell_{i} \in \mathrm{Lab}, L_{i} \subseteq \wp(\mathrm{Op} \times \mathrm{Lab})\right\}$, where Lab is the set of labels, and Op is the set of operators.

An environment relates variables to their values as well as to their atomic data expression. Formally, $\Sigma=D \times V$, where (i) $D: \operatorname{Var} \longrightarrow \wp(\mathbb{D})$ maps local variables in Var to a corresponding adexp, and (ii) $V: \operatorname{Var} \longrightarrow(\mathbb{Z} \cup \mathbb{S}$ ) is the usual environment that tracks value information. The special symbol $\star$ represents data coming from the user input and from the constants of the program. Instead, data coming from the concrete data-store $C$ are represented by $=\left\{\left\langle\ell_{i}, \emptyset\right\rangle: i \in\right.$ $I\} \subseteq \mathbb{D}$ such that $\forall i, j \in I: i \neq j \Rightarrow \ell_{i} \neq \ell_{j}$, and $\ell_{i} \neq \star$.

\subsection{Collecting Semantics}

We extend the notion of atomic data expressions to collect also implicit flows generated by if and while statements. Such flows are treated in the same way as explicit flows by collecting the Boolean expression (bexp) of a conditional or loop statement, and considering it as an adexp with its operators and sources.

Definition 1 (Extended Atomic Data Expressions). We redefine the set of atomic data expressions as: $\mathbb{D}=\left\langle\left\{\left\langle\ell_{i}, L_{i}\right\rangle: i \in I\right\},\left\{\left\langle\ell_{j}, L_{j}\right\rangle: j \in J\right\}\right\rangle$ where $L \subseteq \wp(O p \times L a b)$

An extended atomic data expression can be seen as a pair of two atomic data expressions, where the second one refers to the implicit flows (notice that also a Boolean or relational operator may appear). Formally, $d=\left\langle d^{e}, d^{i}\right\rangle$, where $d^{e}$ and $d^{i}$ correspond to the explicit and implicit flows, respectively. In this way we collect also the Boolean operators.

Consider the conditional statement: if exp then $x=$ case $_{1}$ else $x=$ case $_{2}$. We can interpret each expression as a combination of explicit and implicit flow:

$$
\begin{aligned}
& d_{i f \text { cond }}=\left\langle d^{e}, d^{i}\right\rangle \\
& d_{\text {case }_{1}}=\left\langle d_{\text {case }_{1}}^{e}, d_{\text {case }_{1}}^{i}\right\rangle \\
& d_{\text {case }_{2}}=\left\langle d_{\text {case }_{2}}^{e}, d_{\text {case }_{2}}^{i}\right\rangle
\end{aligned}
$$

Notice that $d^{i}$ in case $_{1}$ and case $_{2}$ expresses only the implicit flow generated inside the two branches of the if statement, and it does not include the implicit flow that comes from the evaluation of the Boolean expression exp. Then, if the case $_{1}$ is chosen, we obtain: $d_{\text {result }}=\left\langle d_{\text {case }_{1}}^{e},\left\{d_{\text {case } e_{1}}^{i} \cup d_{i f}^{e} \cup d_{i f}^{i}\right\}\right\rangle$ where $d_{i f}^{e}$ and $d_{i f}^{i}$ represent the flows in the if condition, both collected in the implicit flow of all the subsequent expressions. The value associated to $x$ after the if-then-else 
statement makes explicit that $x$ has implicit dependence on the sources of the Boolean expression. For instance, if $\exp =y \geq 0$, it will track that the value of $x$ is dependent on the value of $y$.

We denote by $S_{N}: n \exp \times V \rightarrow \mathbb{Z}, S_{S}: \operatorname{sexp} \times V \rightarrow \mathbb{S}$, and $S_{B}: \operatorname{bexp} \times V \rightarrow$ \{true, false\} the standard concrete evaluations of numerical, string, and Boolean expressions. In addition, $S_{L}: \operatorname{lexp} \times \Sigma \rightarrow$ Lab that returns a data label given a label expression. The semantics of expressions on atomic data $S_{A}: \operatorname{sexp} \times \Sigma \rightarrow$ $\wp(\mathbb{D})$ is described in Figure 1. The semantics has been improved w.r.t. [4] with a new operator, checkpwd( $\left.\operatorname{sexp}_{1}, \operatorname{sexp}_{2}\right)$, that returns true if a secret password is equal to a given value. In addition, we track both explicit and implicit flows. In particular, we do not create any implicit flow, and we simply propagate the implicit flows generated by previous expressions: $\left\langle S \llbracket c \rrbracket(a, v),\left\{\left\langle\ell_{j}, L_{j}\right\rangle: j \in J\right\}\right\rangle$.

Similarly, we rewrite the semantics of statements that create implicit flow, that is, the semantics of if and while statements (Fig. 2). The definition of this semantics is split into explicit $\left(S_{e}\right)$ and implicit $\left(S_{i}\right)$ flows. We add the skip statement to handle the exit from a loop statement like the while.

$$
\begin{aligned}
& S_{A} \llbracket x \rrbracket(a, v)=a(x) \\
& S_{A} \llbracket \operatorname{read}(l e x p) \rrbracket(a, v)=\left\{\left\langle S_{L} \llbracket l e x p \rrbracket(a, v), \emptyset\right\rangle\right\} \\
& S_{A} \llbracket \operatorname{encrypt}(\operatorname{sexp}, k) \rrbracket(a, v)=\left\{\left\langle\ell_{1}, L_{1} \cup\left\{\left([\text { encrypt }, k], \ell_{1}\right)\right\}\right\rangle:\left\langle\ell_{1}, L_{1}\right\rangle \in S_{A} \llbracket \operatorname{sexp} \rrbracket(a, s, n)\right\} \\
& S_{A} \llbracket s \rrbracket(a, v)=\{\langle\star, \emptyset\rangle\} \\
& S_{A} \llbracket \operatorname{sexp}_{1} \circ \operatorname{sexp}_{2} \rrbracket(a, v)=\left\{\left\langle\ell_{1}, L_{1} \cup\left\{\left(\circ, \ell_{2}\right)\right\}\right\rangle,\left\langle\ell_{2}, L_{2} \cup\left\{\left(\circ, \ell_{1}\right)\right\}\right\rangle:\right. \\
& \left.\left\langle\ell_{1}, L_{1}\right\rangle \in S_{A} \llbracket \operatorname{sexp}_{1} \rrbracket(a, v),\left\langle\ell_{2}, L_{2}\right\rangle \in S_{A} \llbracket \operatorname{sexp}_{2} \rrbracket(a, v)\right\} \\
& S_{A} \llbracket \operatorname{sub}\left(\operatorname{sexp}, k_{1}, k_{2}\right) \rrbracket(a, v)=\left\{\left\langle\ell_{1}, L_{1} \cup\left\{\left(\left[\operatorname{sub}, k_{1}, k_{2}\right], \ell_{1}\right)\right\}\right\rangle:\left\langle\ell_{1}, L_{1}\right\rangle \in S_{A} \llbracket \operatorname{sexp} \rrbracket(a, v)\right\} \\
& S_{A} \llbracket h a s h(\operatorname{sexp}) \rrbracket(a, v)=\left\{\left\langle\ell_{1}, L_{1} \cup\left(h a s h, \ell_{1}\right)\right\rangle:\left\langle\ell_{1}, L_{1}\right\rangle \in S \llbracket \operatorname{sexp} \rrbracket(a, v)\right\} \\
& S_{A} \llbracket \text { checkpwd }(\operatorname{sexp}, s) \rrbracket(a, v)=\left\{\left\langle\ell_{1}, L_{1} \cup\{(\text { checkpwd, }, \star)\}\right\rangle:\left\langle\ell_{1}, L_{1}\right\rangle \in S_{A} \llbracket \operatorname{sexp}_{1} \rrbracket(a, v)\right\}
\end{aligned}
$$

Fig. 1. Semantics of Expressions on Atomic Data

$$
\begin{aligned}
& S \llbracket x:=\operatorname{sexp} \rrbracket(a, v)=\left(a\left[x \mapsto S_{A} \llbracket \operatorname{sexp} \rrbracket(a, v)\right], v\left[x \mapsto S_{S} \llbracket \operatorname{sexp} \rrbracket(v)\right]\right) \\
& S \llbracket s k i p \rrbracket(a, v)=(a, v) \\
& S \llbracket \operatorname{send}(\operatorname{sexp}) \rrbracket(a, v)=(a, v) \\
& \left.S \llbracket c_{1} ; c_{2} \rrbracket(a, v)=S \llbracket c_{2} \rrbracket\left(S \llbracket c_{1} \rrbracket(a, v)\right)\right) \\
& S \llbracket \text { if } c_{1} \text { then } c_{2} \text { else } c_{3} \rrbracket(a, v)=\left\{\begin{array}{c}
\left\langle S_{e} \llbracket c_{2} \rrbracket(a, v), S_{i} \llbracket c_{2} \rrbracket(a, v) \cup S_{e} \llbracket c_{1} \rrbracket(a, v) \cup S_{i} \llbracket c_{1} \rrbracket(a, v)\right\rangle \\
\text { if } S_{B} \llbracket c_{1} \rrbracket(v) \\
\left\langle S_{e} \llbracket c_{3} \rrbracket(a, v), S_{i} \llbracket c_{3} \rrbracket(a, v) \cup S_{e} \llbracket \neg c_{1} \rrbracket(a, v) \cup S_{i} \llbracket c_{1} \rrbracket(a, v)\right\rangle \\
\text { otherwise }
\end{array}\right. \\
& S \llbracket \text { while } c_{1} \text { do } c_{2} \rrbracket(a, v)=S \llbracket \text { if }\left(c_{1}\right) \text { then }\left(c_{2} ; \text { while } c_{1} \text { do } c_{2}\right) \text { else } \operatorname{skip} \rrbracket(a, v)
\end{aligned}
$$

Fig. 2. Concrete Semantics of Statements

Example. Suppose that $y$ contains a value arising from a data store labeled $\ell_{1}$, while $x$ contains user input. We assume that $>y$.

$$
\begin{aligned}
& 1 \mathrm{y}=\operatorname{read}\left(\ell_{1}\right) \\
& 2 \mathrm{x}=\text { userinput }() ; \\
& 3 \mathrm{w}=9
\end{aligned}
$$




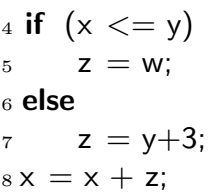

The following are the expressions computed by the collecting semantics, where the subscript represents the code line of the expression:

$$
\begin{aligned}
\mathrm{y}_{1} & :\left\langle\left\{\left\langle\ell_{1}, \emptyset\right\rangle\right\}, \emptyset\right\rangle \\
(\mathrm{x}<=\mathrm{y})_{4} & :\left\langle\left\{\left\langle\ell_{1},\{(>=, \star)\}\right\rangle\right\}, \emptyset\right\rangle \\
\mathrm{y}+3_{7} & :\left\langle\left\{\left\langle\ell_{1},\{(+, \star)\}\right\rangle\right\}, \emptyset\right\rangle \\
\mathrm{z}=\mathrm{y}+3_{7}: & : \underbrace{\left\{\left\langle\ell_{1},\{(+, \star)\}\right\rangle\right\}}_{\text {explicit flow }}, \underbrace{\left\{\left\langle\ell_{1},\{(<, \star)\}\right\rangle\right\}}_{\text {implicit flow }}\rangle \\
\mathrm{x}=\mathrm{x}+\mathrm{z}_{8} & :\left\langle\left\{\left\langle\ell_{1},\{(+, \star)\}\right\rangle\right\},\left\{\left\langle\ell_{1},\{(<, \star)\}\right\rangle\right\}\right\rangle
\end{aligned}
$$

The first three expressions are not affected by implicit flows, so the implicit component in these expressions is $\emptyset$. The second expression refers to the if Boolean expression, and the fourth one refers to the assignment of the else branch, and it takes into account the implicit flow generated by the if statement.

\section{Quantitative Semantics}

In this Section, we extend the collecting semantics by introducing a quantitative notion of information flow.

\subsection{Quantitative Concrete Domain}

We begin by representing values having a binary form to express quantities of information flows. In this way, we adopt a standardized evaluation of quantities coming from different data types.

Definition 2 (Label Dimension). Let $\ell_{i}$ be the label of a location in the datastore. $\omega$ returns the size of the memory location corresponding to the given label, and it is defined by nbit $_{\ell_{i}}:=\omega\left(\ell_{i}\right)$, where nbit is the retrieved dimension in bits.

The value returned $\omega$ depends on the particular type of the datum:

- Numbers: for the sake of simplicity we consider only integer numbers. The number of bits for a label containing such kind of data is: nbits $=\left\lfloor\log _{2}(n)\right\rfloor+1$,

- String: we adopt a simplified representation of characters. In particular, we consider an encoding representing only English alphabet (with uppercase and lowercase letters) and numerical digits. We then have $26+26+10$ elements. Thus this encoding requires 6 bits for each character, and nbits $=6 \times l_{\text {str }}$ where $l_{s t r}$ represents the number of characters of string $s t r$, and

- Boolean: such values can be only 0 or 1 , so they require only 1 bit. 
We now extend the collecting semantics to take into account quantities of information by adding a new expression associated to the extended adexp to the concrete state.

Definition 3 (Quantitative Expression). We define a quantitative expression qadexp as a sequence of pairs of labels associated with quantitative values $\langle\ell, q\rangle$. This collects the quantity of information generated by implicit flows for the given label $\ell$. This sequence is combined with a $d_{q}$ in qadexp in a unique expression as follows:

$$
d:=\left(\left\langle d_{e}, d_{i}\right\rangle, d_{q}\right)
$$

Therefore, we represent data expressions as follows:

$$
\mathbb{D}=\left(\left\langle\left\{\left\langle\ell_{i}, L_{i}\right\rangle: i \in I\right\},\left\{\left\langle\ell_{j}, L_{j}\right\rangle: j \in J\right\}\right\rangle,\left\{\left\langle\ell_{k}, q_{k}\right\rangle: k \in J\right\}\right)
$$

where $\ell_{k}$ are the labels used in statements that generate implicit flow, while $q_{k}$ is the associated quantity of information.

Every single pair $\left\langle\ell_{k}, q_{k}\right\rangle$ tracks the quantity of information that label $\ell_{k}$ potentially released through implicit flow. Notice that the expression $d:=$ $\left(\left\langle d_{e}, d_{i}\right\rangle, d_{q}\right)$ highlights how our analysis is the result of the combination of two approaches, and in particular (i) the first two components expression comes from a qualitative approach to explicit and implicit flows, and (ii) the last one is the result of a quantitative approach.

We define as $Q$ the domain of quantities of information. We are now in position to introduce a function that describes how quantities are collected.

Definition 4 (Quantitative Function $\phi$ ). Let $\phi$ be a function that updates a quantitative value each time the associated label is involved in an implicit flow, such that:

$$
v a l_{\text {post }}^{\ell}:=v a l_{\text {pre }}^{\ell}+\phi_{\text {stm }}(\ell)
$$

where $\phi_{\text {stm }}: \ell \mapsto v a l$ and pre and post refer to the statement (stm) execution.

Quantities are represented as an interval [val,val] were higher and lower bound coincide. This will allow an easier lift to the abstract value. Anyway, for the sake of readability, the singleton interval [ $\mathrm{val}$, val $]$ will be denoted by a single value val.

We have to track quantities of implicit flow generated by if and while statements. Conditional expressions can result only in true and false. Thus, the information obtained from the evaluation of a Boolean condition consists only of one bit [16].

For the sake of simplicity, we consider only the $>$ and $<$ (strict) operators. This avoids problems with equalities $(a==b)$ allowing the collection of only one bit of information for each if statement [2,3]. Figure 3 defines the semantics of conditional expressions. Notice that we do not yet introduce quantities, while we only express how to collect equality in conditional statements using integers instead of Boolean values.. 


$$
\begin{aligned}
S \llbracket \operatorname{sexp} \rrbracket(a, v)= & \left\{\left\langle\ell_{1}, L_{1} \cup\{(>, \star)\}\right\rangle:\left\langle\ell_{1}, L_{1}\right\rangle \in S_{A} \llbracket \operatorname{sexp}_{1} \rrbracket(a, v)\right\} \\
S \llbracket \neg \operatorname{sexp} \rrbracket(a, v)= & \left\{\left\langle\ell_{1}, L_{1} \cup\{(<, \star)\}\right\rangle:\left\langle\ell_{1}, L_{1}\right\rangle \in S_{A} \llbracket \operatorname{sexp}_{1} \rrbracket(a, v)\right\} \\
S \llbracket \exp _{1}>\operatorname{sexp} p_{2} \rrbracket(a, v)= & \left\{\left\langle\ell_{1}, L_{1} \cup\left\{\left(>, \ell_{2}\right)\right\}\right\rangle,\left\langle\ell_{2}, L_{2} \cup\left\{\left(<, \ell_{1}\right)\right\}\right\rangle:\right. \\
& \left.\left\langle\ell_{1}, L_{1}\right\rangle \in S_{A} \llbracket \operatorname{sexp} \exp _{1} \rrbracket(a, v),\left\langle\ell_{2}, L_{2}\right\rangle \in S_{A} \llbracket \operatorname{sexp}_{2} \rrbracket(a, v)\right\} \\
S \llbracket \operatorname{sexp}_{1}<\operatorname{sexp}_{2} \rrbracket(a, v)= & \left\{\left\langle\ell_{1}, L_{1} \cup\left\{\left(<, \ell_{2}\right)\right\}\right\rangle,\left\langle\ell_{2}, L_{2} \cup\left\{\left(>, \ell_{1}\right)\right\}\right\rangle:\right. \\
& \left.\left\langle\ell_{1}, L_{1}\right\rangle \in S_{A} \llbracket \operatorname{sexp} p_{1} \rrbracket(a, v),\left\langle\ell_{2}, L_{2}\right\rangle \in S_{A} \llbracket \operatorname{sexp}_{2} \rrbracket(a, v)\right\}
\end{aligned}
$$

Fig. 3. Concrete Semantics of Conditional Expressions

\subsection{Quantitative Concrete Semantics}

Let $\varphi$ be function that maps variables to quantities $(\varphi: \operatorname{Var} \rightarrow$ qadexp $)$. We now introduce the quantity notion into our quantitative collecting semantics. The initial quantity value is $\phi_{s t m}\left(\ell_{i}\right)=0 \forall i$, and it is modified by $\phi_{s t m}$ only for the statements that generate implicit flow. This means that in the other expressions the $\varphi$ component will be carried as is. The new semantics is defined in Figures 4, 5 and 6.

$$
\begin{aligned}
& S_{A} \llbracket x \rrbracket(a, \varphi, v)=a(x) \\
& S_{A} \llbracket \operatorname{read}(\text { lexp }) \rrbracket(a, \varphi, v)=\left\{\left\langle S_{L} \llbracket l e x p \rrbracket(a, \varphi, v), \emptyset\right\rangle\right\} \\
& S_{A} \llbracket \text { encrypt }(\operatorname{sexp}, k) \rrbracket(a, \varphi, v)=\left\{\left\langle\ell_{1}, L_{1} \cup\left\{\left([\text { encrypt }, k], \ell_{1}\right)\right\}\right\rangle:\right. \\
& \left.\left\langle\ell_{1}, L_{1}\right\rangle \in S_{A} \llbracket \operatorname{sexp} \rrbracket(a, \varphi, s, n)\right\} \\
& S_{A} \llbracket s \rrbracket(a, \varphi, v)=\{\langle\star, \emptyset\rangle\} \\
& S_{A} \llbracket \operatorname{sexp}_{1} \circ \operatorname{sexp}_{2} \rrbracket(a, \varphi, v)=\left\{\left\langle\ell_{1}, L_{1} \cup\left\{\left(\circ, \ell_{2}\right)\right\}\right\rangle,\left\langle\ell_{2}, L_{2} \cup\left\{\left(\circ, \ell_{1}\right)\right\}\right\rangle:\right. \\
& \left\langle\ell_{1}, L_{1}\right\rangle \in S_{A} \llbracket \operatorname{sexp}_{1} \rrbracket(a, \varphi, v) \text {, } \\
& \left.\left\langle\ell_{2}, L_{2}\right\rangle \in S_{A} \llbracket \operatorname{sexp}_{2} \rrbracket(a, \varphi, v)\right\} \\
& S_{A} \llbracket \operatorname{sub}\left(\operatorname{sexp}, k_{1}, k_{2}\right) \rrbracket(a, \varphi, v)=\left\{\left\langle\ell_{1}, L_{1} \cup\left\{\left(\left[s u b, k_{1}, k_{2}\right], \ell_{1}\right)\right\}\right\rangle:\right. \\
& \left.\left\langle\ell_{1}, L_{1}\right\rangle \in S_{A} \llbracket \operatorname{sexp} \rrbracket(a, \varphi, v)\right\} \\
& S_{A} \llbracket h a s h(\operatorname{sexp}) \rrbracket(a, \varphi, v)=\left\{\left\langle\ell_{1}, L_{1} \cup\left(\text { hash }, \ell_{1}\right)\right\rangle:\right. \\
& \left.\left\langle\ell_{1}, L_{1}\right\rangle \in S \llbracket \operatorname{sexp} \rrbracket(a, \varphi, v)\right\} \\
& S_{A} \llbracket \operatorname{checkpwd}(\operatorname{sexp}, s) \rrbracket(a, \varphi, v)=\left\{\left\langle\ell_{1}, L_{1} \cup\{(\operatorname{checkpwd}, \star)\}\right\rangle:\right. \\
& \left.\left\langle\ell_{1}, L_{1}\right\rangle \in S_{A} \llbracket \operatorname{sexp}_{1} \rrbracket(a, \varphi, v)\right\}
\end{aligned}
$$

Fig. 4. Quantitative Semantics of Expressions on Atomic Data

$$
\begin{aligned}
S \llbracket x:=\operatorname{sexp} \rrbracket(a, \varphi, v) & =\left(a\left[x \mapsto S_{A} \llbracket \operatorname{sexp} \rrbracket(a, \varphi, v)\right], v\left[x \mapsto S_{S} \llbracket \operatorname{sexp} \rrbracket(v)\right]\right) \\
S \llbracket \operatorname{skip} \rrbracket(a, \varphi, v) & =(a, \varphi, v) \\
S \llbracket \operatorname{send}(\operatorname{sexp}) \rrbracket(a, \varphi, v) & =(a, \varphi, v) \\
S \llbracket c_{1} ; c_{2} \rrbracket(a, \varphi, v) & \left.=S \llbracket c_{2} \rrbracket\left(S \llbracket c_{1} \rrbracket(a, \varphi, v)\right)\right)
\end{aligned}
$$

Fig. 5. Quantitative Concrete Semantics of Statements 
$S \llbracket$ if $c_{1}$ then $c_{2}$ else $c_{3} \rrbracket(a, v)=$

- if $S_{B} \llbracket c_{1} \rrbracket(v)$ is True then:

$$
\begin{gathered}
\text { let }\left(a^{\prime}, \varphi^{\prime}, v^{\prime}\right)=\left\langle S_{e} \llbracket c_{2} \rrbracket(a, \varphi, v),\right. \\
\left.\quad S_{i} \llbracket c_{2} \rrbracket(a, \varphi, v) \cup S_{e} \llbracket c_{1} \rrbracket(a, \varphi, v) \cup S_{i} \llbracket c_{1} \rrbracket(a, \varphi, v)\right\rangle \\
\text { in }\left(a^{\prime}, \varphi^{\prime}\left[\left(\ell_{i}, q_{i}\right) /\left(\ell_{i}, q_{i}+\phi_{s t m}\left(c_{1}\right)\right): \ell_{i} \in \operatorname{src}\left(c_{1}\right)\right], v^{\prime}\right)
\end{gathered}
$$

- otherwise:

$$
\begin{gathered}
\text { let }\left(a^{\prime}, \varphi^{\prime}, v^{\prime}\right)=\left\langle S_{e} \llbracket c_{3} \rrbracket(a, \varphi, v),\right. \\
\left.\quad S_{i} \llbracket c_{3} \rrbracket(a, \varphi, v) \cup S_{e} \llbracket \neg c_{1} \rrbracket(a, \varphi, v) \cup S_{i} \llbracket c_{1} \rrbracket(a, \varphi, v)\right\rangle \\
\text { in }\left(a^{\prime}, \varphi^{\prime}\left[\left(\ell_{i}, q_{i}\right) /\left(\ell_{i}, q_{i}+\phi_{s t m}\left(c_{1}\right)\right): \ell_{i} \in \operatorname{src}\left(c_{1}\right)\right], v^{\prime}\right)
\end{gathered}
$$

where $\phi_{s t m}$ is the quantitative function in Def. 4

$S \llbracket$ while $c_{1}$ do $c_{2} \rrbracket(a, \varphi, v)=$

$$
S \llbracket \text { if }\left(c_{1}\right) \text { then }\left(c_{2} ; \text { while } c_{1} \text { do } c_{2}\right) \text { else skip } \rrbracket(a, \varphi, v)
$$

Fig. 6. Quantitative Concrete Semantics of Control Statements

\section{$5 \quad$ Abstract Semantics}

We now extend the abstract semantics proposed by Cortesi et al. [4] to implicit flows and a quantitative analysis. Our abstract semantics is parameterized by a value domain $V^{a}$, and a label abstraction. First of all, we need to define a computable abstraction of quantities.

Definition 5 (Quantity Value Abstraction). The quantity associate with label expressions is an interval. Each label $\ell^{a}$ is associated to an interval of quantities where the lower and the upper bounds are the minimum and the maximum quantities of information that can be released through the implicit flow for that specific label. Therefore, the abstract qadexp is defined as $\left\langle\ell_{k}^{a}, q_{k_{\text {min }}}^{a}, q_{k_{\text {max }}}^{a}\right\rangle$.

If we have unbounded quantities, the analysis reveals a complete leakage of the associated label. In this case, the upper bound of the intervals is unbounded. Instead, for the lower bound the minimum quantity is zero.

\subsection{Atomic Data Abstraction Extension}

We now define the atomic data abstraction extended for handling implicit flows and quantities.

Definition 6 (Abstract Extended Atomic Data and Quantities). Let us consider a set of atomic data and quantity values. We define abstract elements as $\left(\left\langle\left\{\left\langle\ell_{w}^{a}, L_{w}^{a \sqcap}, L_{w}^{a \sqcup}\right\rangle: w \in W\right\},\left\{\left\langle\ell_{z}^{a}, L_{z}^{a \sqcap}, L_{z}^{a \sqcup}\right\rangle: z \in Z\right\}\right\rangle,\left\{\left\langle\ell_{g}^{a}, q_{g}^{a \sqcap}, q_{g}^{a \sqcup}\right\rangle: g \in Z\right\}\right)$ 
where:

- $\ell_{w}^{a}$ is an element that abstracts labels in Lab to track explicit flow,

- $\ell_{z}^{a}$ and $\ell_{g}^{a}$ are elements that abstract labels in Lab to track implicit flow,

- $L_{w}^{a \sqcap}=\left\{\left(o p_{i w}^{a}, \ell_{i w}^{a}\right): i \in I\right\}$ and $L_{z}^{a \sqcap}=\left\{\left(o p_{j z}^{a}, \ell_{j z}^{a}\right): j \in J\right\}$ represent the under-approximation of $\ell_{w}^{a}$ and $\ell_{z}^{a}$ with labels abstracted by $\ell_{i w}^{a}$ and $\ell_{j z}^{a}$, and track explicit and implicit flows, respectively,

- $L_{w}^{a \sqcup}=\left\{\left(o p_{i w}^{a}, \ell_{i w}^{a}\right): i \in I^{\prime}\right\}$ and $L_{z}^{a \sqcup}=\left\{\left(o p_{j z}^{a}, \ell_{j z}^{a}\right): j \in J^{\prime}\right\}$ represent the over-approximation of $\ell_{w}^{a}$ and $\ell_{z}^{a}$ with labels abstracted by $\ell_{i w}^{a}$ and $\ell_{j z}^{a}$, and track explicit and implicit flows, respectively,

- $L_{w}^{a \sqcap} \subseteq L_{w}^{a \sqcup}$ and $L_{z}^{a \sqcap} \subseteq L_{z}^{a \sqcup,}$

- $q_{g}^{a}$ is an element that abstract quantites associated to a $\ell_{g}^{a}$ element,

- $q_{k g}^{a \sqcap}: k \in J$ is an under-approximation of the interval of possible quantities of information associated to $\ell_{g}^{a}$ with values represented by $q_{k g}^{a}$,

- $q_{k g}^{a \sqcup}: k \in J^{\prime}$ is an over-approximation of the interval of possible quantities of information associated to $\ell_{g}^{a}$ with values represented by $q_{k g}^{a}$, and

$-q_{g}^{a \sqcap} \leqslant q_{g}^{a \sqcup}$.

As a corollary, we define the source set of an atomic datum $\left\langle\left\{\ell_{w}^{a}: w \in W\right\},\left\{\ell_{z}^{a}\right.\right.$ : $z \in Z\}\rangle$ expressed as $\operatorname{src}(d)$.

Although we inherit the abstraction and concretization functions for the explicit flows [4], we have to extend them to handle quantities.

Definition 7 (Quantitative Abstraction Function). We denote by $\alpha_{\mathrm{Q}}$ the abstraction function that given a set $\left\{\left(\ell_{k}, q_{k}\right): k \in J\right\}$ returns $\left\{\left(\alpha_{\mathrm{Lab}}\left(\ell_{k}\right), q_{k}^{a \sqcap}, q_{k}^{a \sqcup}\right): k \in J\right\}$, where $q_{k}^{a \sqcap}, q_{k}^{a \sqcup}$ represent the bounds of the interval that approximates all possible quantitative values in the abstract domain $Q^{a}$.

Definition 8 (Quantitative Abstraction Function for Atomic Data). Given a concrete atomic datum $d=\left(\left\langle\left\{\left\langle\ell_{i}, L_{i}\right\rangle: i \in I\right\},\left\{\left\langle\ell_{j}, L_{j}\right\rangle: j \in\right.\right.\right.$ $\left.J\}\rangle,\left\{\left\langle\ell_{k}, q_{k}\right\rangle: k \in J\right\}\right)$, we define an abstraction function $\alpha: \wp(\mathbb{D}) \longrightarrow \mathbb{A D}$ as:

$$
\begin{aligned}
\alpha_{s}(d)= & \left(\left\langle\left\{\left\langle\alpha_{\mathrm{Lab}}\left(\ell_{i}\right), \alpha_{\mathrm{Lab}}\left(L_{i}\right), \alpha_{\mathrm{Lab}}\left(L_{i}\right)\right\rangle: i \in I\right\},\right.\right. \\
& \left.\left\{\left\langle\alpha_{\mathrm{Lab}}\left(\ell_{j}\right), \alpha_{\mathrm{Lab}}\left(L_{j}\right), \alpha_{\mathrm{Lab}}\left(L_{j}\right)\right\rangle: j \in J\right\}\right\rangle, \\
& \left.\left\{\left\langle\alpha_{\mathrm{Lab}}\left(\ell_{k}\right), \alpha_{\mathrm{Q}}\left(q_{k}\right)\right\rangle: k \in J\right\}\right)
\end{aligned}
$$

The abstraction function is then extended to sets by computing the upper bound of the point-wise application of $\alpha_{s}$ to all the elements of the given set.

\subsection{Abstract Semantics of Statements}

Expressions are abstracted via an abstract data label and an abstract value ( $A D^{a}$ and $V^{a}$, respectively). In Figure 7 the abstract semantics of statements taking into account implicit flows is defined Then, in Figure 8 this semantics is extended with the quantitative dimension. We omit here the abstract semantics of expressions, as it does not generate any implicit flow, and we refer the interested reader to Cortesi et. al [4] for more details. 


$$
\begin{aligned}
S^{a} \llbracket x:=\operatorname{sexp} \rrbracket\left(a^{a}, v^{a}\right) & =\left(a^{a}, S_{v}^{a} \llbracket x:=\operatorname{sexp} \rrbracket\left(v^{a}\right)\right) \\
S^{a} \llbracket \operatorname{skip} \rrbracket\left(a^{a}, v^{a}\right) & =\left(a^{a}, v^{a}\right) \\
S^{a} \llbracket \operatorname{send}(\operatorname{sexp}) \rrbracket\left(a^{a}, v^{a}\right) & =\left(a^{a}, v^{a}\right) \\
S^{a} \llbracket c_{1} ; c_{2} \rrbracket\left(a^{a}, v^{a}\right) & =S^{a} \llbracket c_{2} \rrbracket\left(S^{a} \llbracket c_{1} \rrbracket\left(a^{a}, v^{a}\right)\right)
\end{aligned}
$$

$S^{a} \llbracket$ if $c_{1}$ then $c_{2}$ else $c_{3} \rrbracket\left(a^{a}, v^{a}\right)=$

$$
\begin{array}{r}
\left\langle S_{e}^{a} \llbracket c_{2} \rrbracket\left(a^{a}, S_{e}^{a} \llbracket c_{1} \rrbracket\left(v^{a}\right)\right) \sqcup S_{e}^{a} \llbracket c_{3} \rrbracket\left(a^{a}, S_{e}^{a} \llbracket \neg c_{1} \rrbracket\left(v^{a}\right)\right),\right. \\
S_{i}^{a} \llbracket c_{2} \rrbracket\left(a^{a}, S_{e}^{a} \llbracket c_{1} \rrbracket\left(v^{a}\right)\right) \sqcup S_{e}^{a} \llbracket c_{1} \rrbracket\left(a^{a}, v^{a}\right) \sqcup \\
S_{i}^{a} \llbracket c_{1} \rrbracket\left(a^{a}, v^{a}\right) \sqcup S_{i}^{a} \llbracket c_{3} \rrbracket\left(a^{a}, S_{e}^{a} \llbracket \neg c_{1} \rrbracket\left(v^{a}\right)\right) \sqcup \\
\left.S_{e}^{a} \llbracket \neg c_{1} \rrbracket\left(a^{a}, v^{a}\right) \sqcup S_{i}^{a} \llbracket c_{1} \rrbracket\left(a^{a}, v^{a}\right)\right\rangle
\end{array}
$$

$S^{a} \llbracket$ while $c_{1}$ do $c_{2} \rrbracket(a, v)=$

fix $\left(S^{a} \llbracket\right.$ if $\left(c_{1}\right)$ then $\left(c_{2} ;\right.$ while $c_{1}$ do $\left.c_{2}\right)$ else skip $\left.\rrbracket\left(a^{a}, v^{a}\right)\right)$

Fig. 7. Abstract Semantics of Statements

$$
\begin{aligned}
S^{a} \llbracket x:=\operatorname{sexp} \rrbracket\left(a^{a}, \varphi^{a}, v^{a}\right) & =\left(a^{a}\left[x \mapsto S_{A}^{a} \llbracket \operatorname{sexp} \rrbracket\left(a^{a}, \varphi^{a}, v^{a}\right)\right], v^{a}\left[x \mapsto S_{S}^{a} \llbracket \operatorname{sexp} \rrbracket\left(v^{a}\right)\right]\right) \\
S^{a} \llbracket \operatorname{skip} \rrbracket\left(a^{a}, \varphi^{a}, v^{a}\right) & =\left(a^{a}, \varphi^{a}, v^{a}\right) \\
S^{a} \llbracket \operatorname{send}(\operatorname{sexp}) \rrbracket\left(a^{a}, \varphi^{a}, v^{a}\right) & =\left(a^{a}, \varphi^{a}, v^{a}\right) \\
S^{a} \llbracket c_{1} ; c_{2} \rrbracket\left(a^{a}, \varphi^{a}, v^{a}\right) & =S^{a} \llbracket c_{2} \rrbracket\left(S^{a} \llbracket c_{1} \rrbracket\left(a^{a}, \varphi^{a}, v^{a}\right)\right)
\end{aligned}
$$

$S^{a}$ 【if $c_{1}$ then $c_{2}$ else $c_{3} \rrbracket\left(a^{a}, \varphi^{a}, v^{a}\right)=$

$$
\begin{aligned}
\operatorname{let}\left(a^{\prime a}, \varphi^{\prime a}, v^{\prime a}\right)= & \left\langle S_{e}^{a} \llbracket c_{2} \rrbracket\left(a^{a}, \varphi^{a}, S_{e}^{a} \llbracket c_{1} \rrbracket\left(v^{a}\right)\right) \sqcup S_{e}^{a} \llbracket c_{3} \rrbracket\left(a^{a}, \varphi^{a}, S_{e}^{a} \llbracket \neg c_{1} \rrbracket\left(v^{a}\right)\right),\right. \\
& S_{i}^{a} \llbracket c_{2} \rrbracket\left(a^{a}, \varphi^{a}, S_{e}^{a} \llbracket c_{1} \rrbracket\left(v^{a}\right)\right) \sqcup S_{e}^{a} \llbracket c_{1} \rrbracket\left(a^{a}, \varphi^{a}, v^{a}\right) \sqcup \\
& S_{i}^{a} \llbracket c_{1} \rrbracket\left(a^{a}, \varphi^{a}, v^{a}\right) \sqcup S_{i}^{a} \llbracket c_{3} \rrbracket\left(a^{a}, \varphi^{a}, S_{e}^{a} \llbracket \neg c_{1} \rrbracket\left(v^{a}\right)\right) \sqcup \\
& \left.S_{e}^{a} \llbracket \neg c_{1} \rrbracket\left(a^{a}, \varphi^{a}, v^{a}\right) \sqcup S_{i}^{a} \llbracket c_{1} \rrbracket\left(a^{a}, \varphi^{a}, v^{a}\right)\right\rangle
\end{aligned}
$$

in $\left(a^{\prime a}, \varphi^{\prime a}\left[\left(\ell_{i}^{a}, q_{i}^{a \sqcap}, q_{i}^{a \sqcup}\right) /\left(\ell_{i}^{a}, q_{i}^{a \sqcap}+\phi_{s t m}^{a \sqcap}\left(c_{1}\right), q_{i}^{a \sqcup}+\phi_{s t m}^{a \sqcup}\left(c_{1}\right)\right): \ell_{i}^{a} \in \operatorname{src}\left(c_{1}\right)\right], v^{\prime a}\right)$

where $\phi_{s t m}$ is the quantitative function in Def. 4

$S^{a} \llbracket$ while $c_{1}$ do $c_{2} \rrbracket\left(a^{a}, \varphi^{a}, v^{a}\right)=$ fix $\left(S^{a} \llbracket\right.$ if $\left(c_{1}\right)$ then $\left(c_{2} ;\right.$ while $c_{1}$ do $\left.c_{2}\right)$ else skip $\left.\rrbracket\left(a^{a}, \varphi^{a}, v^{a}\right)\right)$

Fig. 8. Quantitative Abstract Semantics of Statements

We need to abstract the number of iterations of a while loop to precisely approximate the quantity of information leaked by a loop. Our approach is composed by two steps: a while interval analysis approximating the number of iterations, followed by an extended adexp collection with quantitative values.

Step (a): while Interval Analysis. We add a counter initialized to 0 at the beginning, and we increment it by one at each loop iteration. In this way, we can apply a standard interval analysis to infer an upper bound on the number of iterations of a loop. 
Example Consider the following program, where $i$ represents the counter we added:

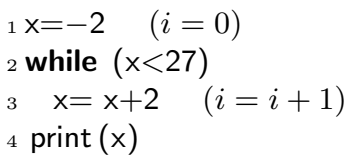

At the end of the analysis, the interval domain can infer $i \mapsto[0 . .15]$ (e.g., by applying a narrowing operator after widening [5]). The upper bound of the interval of variable $i$ returns the number of iterations of the loop, that is, 15 . Then the upper bound on the number of iterations 15 becomes [1,4], where 4 are the number of bits leaked in 15 iterations.

Step (b): Extended Adexp Collection with Quantitative Value. At the end of the while interval analysis, we apply a quantitative value analysis. Example Consider the following example:

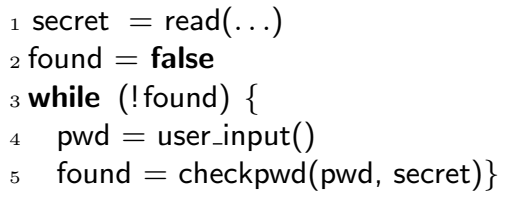

At the end of the first iteration of the analysis of the loop, we obtain:

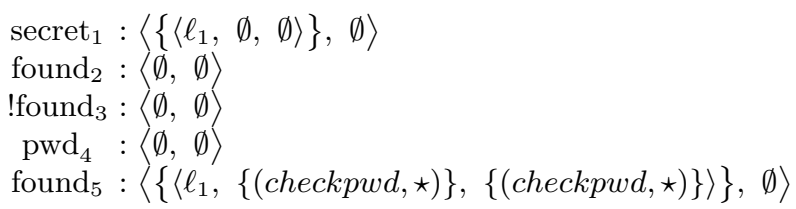

Notice that no quantitative information has yet been released. In fact, the Boolean condition of the while loop is checked against a constant value (false) during the first iteration. However, found might be still false, and the loop would be iterated another time. Then, starting from the second iteration the implicit flow will contain the new definition of the variable found, thus each expression inside the scope of the while will be:

$$
\left\langle\{\ldots \text { explicit flow... }\},\left\{\left\langle\ell_{1},\{(\text { checkpwd }, \star)\},\{(\text { checkpwd }, \star),(<, \star),(>, \star\}\rangle\right\}\right\rangle\right.
$$

Notice that the function checkpw $\left(p_{1}, p_{2}\right)$ returns a Boolean value, so one bit. This means that we are accumulating a bit of information at each iteration. 
As soon as the implicit flow comes into the picture, we have to consider the quantity interval computed in step $(a)$ :

$$
\begin{gathered}
\left\{\left\langle\{\ldots \text { explicit flow... }\},\left\{\left\langle\ell_{1},\{(\text { checkpwd, }, *)\},\{(\text { checkpwd, }, \star),(<, \star),(>\right.\right.\right.\right. \\
\left., \star\}\rangle\}\rangle,\left\langle\ell_{1}, 1,4\right\rangle\right\}
\end{gathered}
$$

If inside a while loop there is an obfuscating operator (e.g., encryption or hashing) applied to confidential data, we need to know the quantity of information that is released by the operator. For instance, in the previous example the operator checkpwd $\left(p_{1}, p_{2}\right)$ checks if the password given by the user is correct, and it returns a Boolean value. Thus the analysis accumulates a single bit at each iteration, and the quantitative value will depend on the number of iterations. However, other operators might release more information. In this case, we compute the product of the number of iterations and the released bits.

\section{Applications}

In this Section, we discuss the results of our analysis of some examples listed in the DroidBench application set [20], created by the Secure Software Engineering group of the Technische Universität Darmstadt. This set is open source, and it is a standard benchmark to test static and dynamic analyses. We chose the examples that specifically deal with implicit flows.

An interesting comparison can be made with the work by Tripp and Rubin [25], as they also used DroidBench as testing environment. Their approach performs very well on the whole test set (also with respect to other tools, like TaintDroid), but it suffers from false negatives in case of implicit flows. Our analysis instead allows to cope with these particular cases, for instance the ones due to custom transformations of private data in the ImplicitFlow1 test program. This is because we adopted a different approach, that instead of looking for privacy sinks, observes the whole flow of confidential data and operations applied to them.

For the sake of readability, we simplified some library functions, and we added some semantic rules to support some primitive functions contained in these examples, and that were not part of the minimal language we adopted in our formalization. For each example, we describe the results of the collecting semantics on a particular concrete state, and we then perform a two-stages static analysis. First we illustrate the results without the quantitative component qadexp, and we then discuss the results of the quantitative semantics.

\subsection{ImplicitFlow1}

The first example is an application that reads the device identifier (IMEI), obfuscates it, and then leaks it. The obfuscation can be performed with two functions with two different obfuscation powers (namely, low and high). 


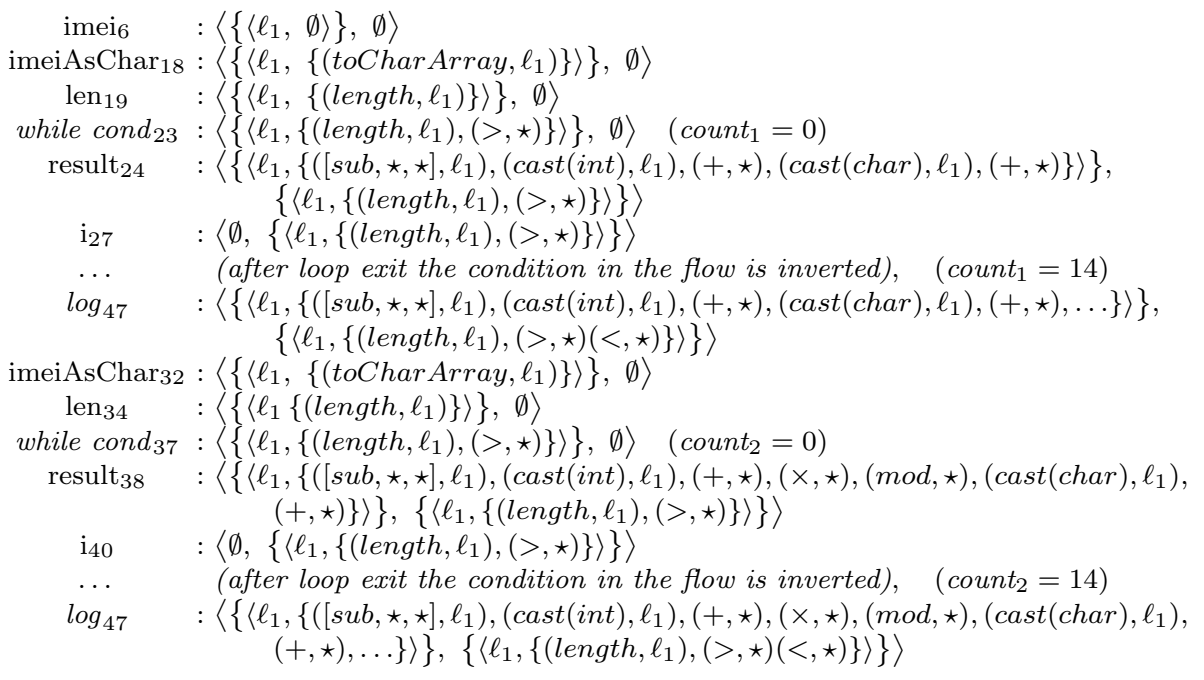

As we can see from the concrete analysis, there is no noticeable difference between the two functions. Indeed, both example apply these function inside a loop, and in both the conditional expressions depend on the dimension of the secret label.

Abstract Analysis. The main differences between the concrete and abstract semantics consist in (i) considering when the loop condition holds and does not (unlike the concrete semantics that always knows a precise value), and (ii) the application of the interval analysis to over-approximate the number of iterations. Notice that since the dimension of the IMEI does not change, the corresponding label can be abstracted with full precision. The same applies to its dimension.

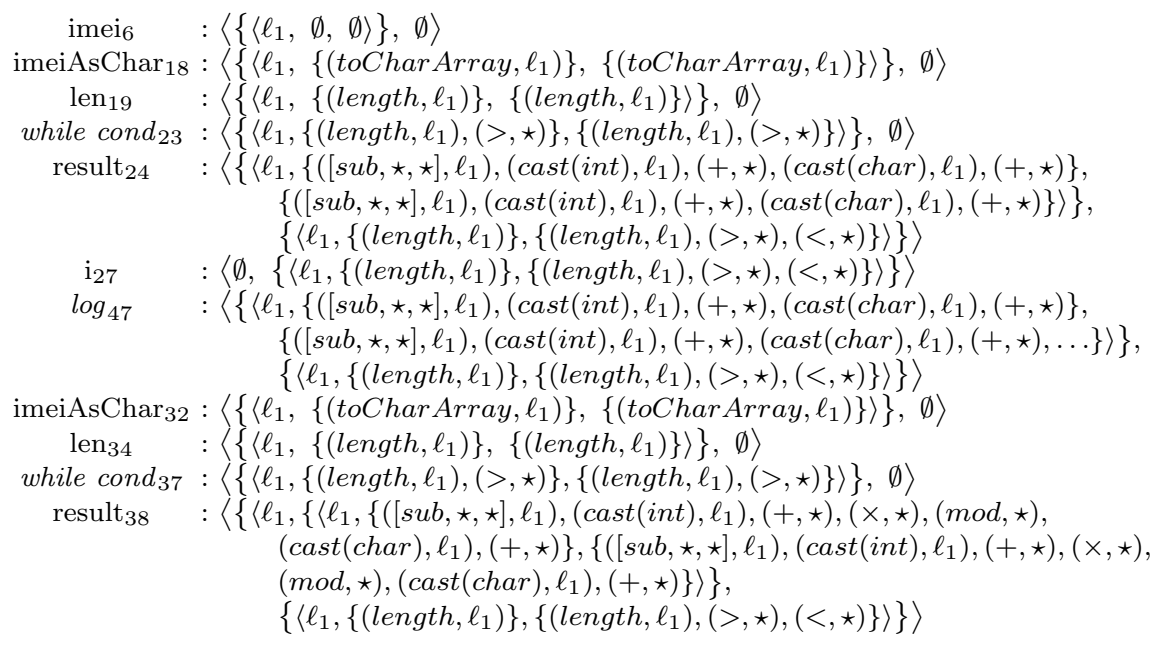




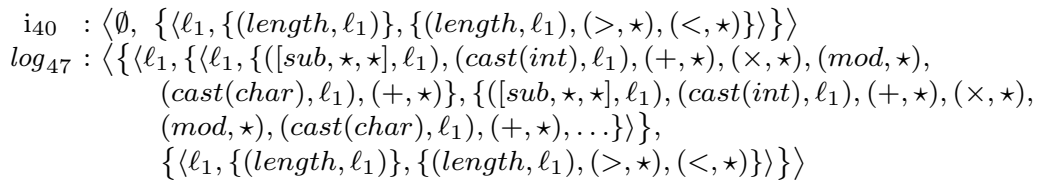

Quantitative Analysis. The interval analysis approximates the minimum and maximum number of iterations of the loop. Through these bounds, we compute an interval of quantities, that infers the minimum and the maximum amount of information revealed through implicit flows. However, in this particular example the number of iterations can be precisely inferred because it is performed on a fixed value (the dimension of the IMEI ). Thus, we infer that the loop is iterated 14 times. Each iteration of the loop leaks one bit of information. We now infer the number of bits using the method described in the Section 5.2:

$$
\text { quantity }=\left\lfloor\log _{2}\left(n \_ \text {iterations }\right)\right\rfloor+1=4 \text { bits }
$$

So, in both loops, the qadexp is $\left\langle\ell_{1}, 4,4\right\rangle$.

\subsection{ImplicitFlow2}

In this example, the user has to type a password. Then, this password is compared to the correct one, that comes from the data-store. After this evaluation, a message is saved to a log file. This operation leaks information through an implicit flow, since the logged message depends on the correctness of the password.

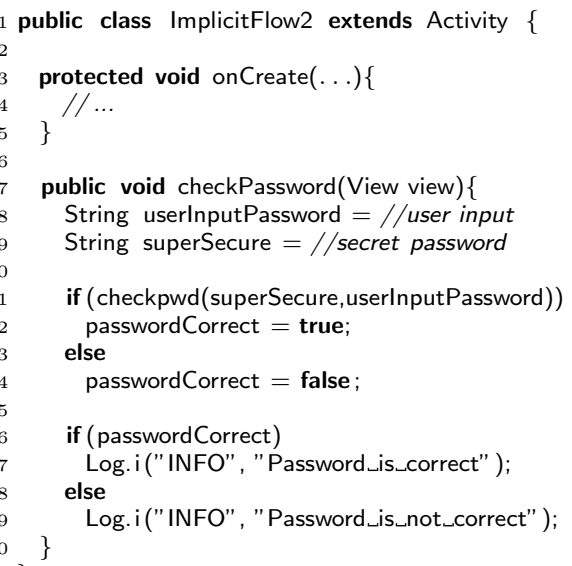

Concrete Analysis. We reuse the semantics of Log defined for the previous example $\left(S_{A} \llbracket \log (\operatorname{sexp}) \rrbracket(a, v)=(a, v)\right)$. The abstract semantics is similar. We now shows the results of the concrete semantics, assuming that the password provided by the user is not correct. The concrete data-store contains only the label that corresponds to the variable superSecure, that is, $\left\langle\ell_{1}, \emptyset\right\rangle$. 


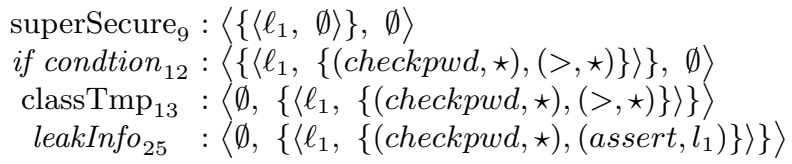

Abstract Analysis. We adopt the same label abstraction.

superSecure $_{9}:\left\langle\left\{\left\langle\ell_{1}, \emptyset, \emptyset\right\rangle\right\}, \emptyset\right\rangle$

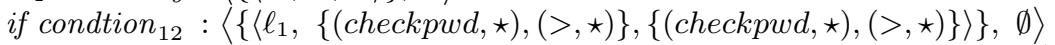

classTmp $_{13,14}:\left\langle\emptyset,\left\{\left\langle\ell_{1},\{(\right.\right.\right.$ checkpwd,,$\star)\},\{($ checkpwd,$\left.\left.\left.\star),(>, \star),(<, \star)\}\right\rangle\right\}\right\rangle$

leakInfo $_{25,31}:\left\langle\emptyset,\left\{\left\langle\ell_{1},\{(\right.\right.\right.$ checkpwd,$\left.\left.\star)\}\right\rangle\right\},\left\{\left\langle\ell_{1},\{(\right.\right.$ checkpwd,$\left.\left.\left.\star),(>, \star),(<, \star)\}\right\rangle\right\}\right\rangle$

Quantitative Analysis. In this example, there is only one if statement that generates implicit flow. This statement exposes 1 bit of quantity of information:

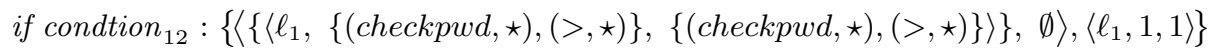

This value will remain the same in all the following qadexps.

\subsection{Discussion}

As emphasized by the examples above, the adoption of a quantitative analysis allows the evaluation of quantities of confidential data that might be released. In ImplicitFlow1, the analysis tells that an implicit flows exists, that confidential labels are contained in it, and it also estimate the potential quantities of data released. This quantity is calculated by the operations implemented in the code, so by the operations that obfuscate the confidential label. The application of given policies [4] will allow to establish whether the released quantities are allowed or not. Any considerations about the safeness of the analyzed application are thus referred to the type of applied policy. In particular, as for ImplicitFlow1, we are able to calculate how much the while loop affects the produced quantity of data. Indeed, it allows to understand that, given a fixed (and possibly low) number of loop iterations, the quantity of confidential data that might be released will not be high. In conclusion, this analysis is not only capable of locating implicit flows, but also to evaluate their importance. In fact, if the released values will be low, with respect to a given policy, the implicit flow will be negligible.

\section{Conclusions}

In this paper, we extended the framework for tracking explicit flows introduced in [4] with respect to implicit flows and quantitative analysis, showing the effectiveness of this approach on significant examples. This framework can support complex hybrid policies, i.e. policies that can grant both qualitative and quantitative thresholds.

Acknowledgments. Work partially supported by the Italian MIUR project "Security Horizons". 


\section{References}

1. Arzt, S., Rasthofer, S., Fritz, C., Bodden, E., Bartel, A., Klein, J., Le Traon, Y., Octeau, D., McDaniel, P.: Flowdroid: Precise context, flow, field, object-sensitive and lifecycle-aware taint analysis for android apps. SIGPLAN Not. 49(6), 259-269 (2014)

2. Clark, D., Hunt, S., Malacaria, P.: Quantitative analysis of the leakage of confidential data. Electronic Notes in Theoretical Computer Science 59(3), 1-14 (2002). Quantitative Aspects of Programming Languages (Satellite Event for PLI 2001)

3. Clark, D., Hunt, S., Malacaria, P.: Quantified interference for a while language. Electr. Notes Theor. Comput. Sci. 112, 149-166 (2005)

4. Cortesi, A., Ferrara, P., Pistoia, M., Tripp, O.: Datacentric semantics for verification of privacy policy compliance by mobile applications. In: D'Souza, D., Lal, A., Larsen, K.G. (eds.) VMCAI 2015. LNCS, vol. 8931, pp. 61-79. Springer, Heidelberg (2015)

5. Cortesi, A., Zanioli, M.: Widening and narrowing operators for abstract interpretation. Computer Languages, Systems \& Structures 37(1), 24-42 (2011)

6. Cousot, P., Cousot, R.: Abstract interpretation: a unified lattice model for static analysis of programs by construction or approximation of fixpoints. In: Conference Record of the Fourth ACM SIGPLAN-SIGACT Symposium on Principles of Programming Languages, pp. 238-252. ACM Press (1977)

7. Denning, D.E.: A lattice model of secure information flow. Communications of the ACM 19, 236-243 (1976)

8. Enck, W., Gilbert, P., Han, S., Tendulkar, V., Chun, B.-G., Cox, L.P., Jung, J., McDaniel, P., Sheth, A.N.: Taintdroid: An information-flow tracking system for realtime privacy monitoring on smartphones. ACM Trans. Comput. Syst. 32(2), 5:1-5:29 (2014)

9. Enck, W., Octeau, D., Mcdaniel, P., Chaudhuri, S.: A study of android application security. In: Proc. USENIX Security Symposium (2011)

10. Fritz, C., Arzt, S., et al.: Highly precise taint analysis for android application. Technical report, EC SPRIDE Technical Report TUD-CS-2013-0113 (2013). http:// www.bodden.de/pubs/TUD-CS-2013-0113.pdf

11. Hammer, C., Snelting, G.: Flow-sensitive, context-sensitive, and object-sensitive information flow control based on program dependence graphs. International Journal of Information Security 8, 399-422 (2009)

12. Hornyack, P., Han, S., Jung, J., Schechter, S., Wetherall, D.: These aren't the droids you're looking for: retrofitting android to protect data from imperious applications. In: Proc. 18th ACM Conf. on Computer and Communications Security, pp. 639-652. ACM, New York (2011)

13. International Data Corporation. Worldwide Quarterly Mobile Phone Tracker 3q14. http://www.idc.com/tracker/showproductinfo.jsp?prod-id=37 (accessed January 2015)

14. Lowe, G.: Quantifying information flow In: Proc. IEEE Computer Security Foundations Workshop, pp. 18-31 (2002)

15. McAfee Labs. Threats Report. http://www.mcafee.com/ca/resources/reports/ rp-quarterly-threat-q3-2014.pdf (accessed December 2014)

16. Mccamant, S., Ernst, M.D.: A simulation-based proof technique fordynamic information flow (2007)

17. McCamant, S., Ernst, M.D.: Quantitative information flow as network flow capacity. SIGPLAN Not. 43(6), 193-205 (2008) 
18. Rasthofer, S., Arzt, S., Lovat, E., Bodden, E.: Droidforce: enforcing complex, datacentric, system-wide policies in android. In: Proceedings of the 9th International Conference on Availability, Reliability and Security (ARES). IEEE, September 2014

19. Russo, A., Sabelfeld, A., Li, K.: Implicit flows in malicious and nonmalicious code. In: Logics and Languages for Reliability and Security. NATO Science for Peace and Security Series, vol. 25, pp. 301-322. IOS Press (2010)

20. Secure Software Engineering Group - Ec Spride. DroidBench. http://sseblog. ec-spride.de/tools/droidbench/ (accessed February 2015)

21. Smith, G.: Principles of secure information flow analysis. In: Christodorescu, M., et al. (eds.) Malware Detection. Advances in Information Security, vol. 27, pp. 291-307. Springer (2007)

22. Sridharan, M., Artzi, S., Pistoia, M., Guarnieri, S., Tripp, O., Berg, R.: F4f: taint analysis of framework-based web applications. In: OOPSLA. ACM (2011)

23. Tripp, O., Ferrara, P., Pistoia, M.: Hybrid security analysis of web javascript code via dynamic partial evaluation. In: Proc. of the 2014 Int. Symposium on Software Testing and Analysis, ISSTA 2014, pp. 49-59. ACM, New York (2014)

24. Tripp, O., Pistoia, M., Fink, S.J., Sridharan, M., Weisman, O.: Taj: effective taint analysis of web applications. In: ACM PLDI, pp. 87-97. ACM (2009)

25. Tripp, O., Rubin, J.: A bayesian approach to privacy enforcement in smartphones. In: USENIX Security (2014) 